\title{
21. A Clinical Survey of Abscess of the Brain
}

\author{
Special Reference to Angiographic Classification \\ and Treatment of Abscess of the Brain \\ Mizuo Kagawa Toshio Beppu, Nobuko Ogawa, \\ Toyoaki Shinohara and Koichi Kitamura \\ Department of Neurosurgery, Tokyo Women's Medical College
}

After antibiotic therapy had been developed, abscess of the brain seems to be under control although mortality and morbidity of brain abscess are still high without adequate early diagnosis and treatment.

With regard to early diagnosis and treatment, several methods have been established. The most powerful diagnostic aids were angiography, brain scan and EEG. Brain scan and EEG, however, could not manifest developmental process of the capsule of brain abscess.

In this paper, we intended to elicit the angiographical developmental process of the capsule of brain abscess and to establish adequate treatment according to angiographic classification of brain abscess.

In our series of 23 cases, clinical studies including angiography, clinical symptoms and pathohistology of operative material and necropsy material are surveyed.

With regards to angiographic and pathohistological classification, abscess of the brain is devided into 3 stages. The classification are as follows:

stage 1: acute stage

stage 2: subcute stage

stage 3: chronic stage

Each 3 stage has specific angiographic findings, therefore repeated angiographical study shows talkatively a clinical pathohistological process of brain abscess, and peri-capsular inflammatory process. The treatment of abscess of the brain are still in debate.

Our program of treatment of brain abscess are as follows. As to the diagnostic procedure, repeated angiography is supreme and angiographical classification should be carried out. In case with stage 1 of angiographical classification; conservative treatments including adequate systemic antibiotics and intravenous administration of gamma globulin, occasional use of steroid hormone and anti-hypotensive drugs such as mannitol and intrathecal administration of antibiotics.

In case of stage 2, under systemic administration of antibiotics and intravenous gamma gulobulin, burr hole is opened and multiaspiration should be carried out and antibiotics and radio-opaque material are given into abscess cavity and total extracapsular extirapation of abscess should be performed in angiographical classfication of stage 3 . 